\title{
Theoretical and Experimental Studies on the Resistivity of Molten Alloys*
}

\author{
By Sakae Takeuchi** and Hirohisa Endo**
}

\begin{abstract}
The resistivity of binary molten alloys were studied theoretically and experimentally.
The resistivity was given theoretically as a function of compressibility and the heat of mixing of molten alloys from the viewpoint that the temperature dependence and the concentration dependence of the resistivity were contributed from density fluctuation and concentration fluctuation due to the the:mal motion in the random distribution of atoms in the alloys.

In dilute alloys Linde's law and Mathiesen's law were deduced and confirmed experimentally in some alloys, excepting those of divalent metals. And it was deduced that the resistivity of molten alloys depended linearly upon the concentration in the case of alloys of component metals with the same atomic valency, and depended parabolically upon the concentration in the case of alloys with different valencies. Experimentally in $\mathrm{Bi}-\mathrm{Sb}$ and $\mathrm{Pb}-\mathrm{Sn}$ alloys, the concentration dependence of the resistivity was shown to be nearly linear while in $\mathrm{Bi}$-In and $\mathrm{Bi}$-Sn alloys, it was confirmed to be parabolic. (Received February 21, 1962)
\end{abstract}

\section{Introduction}

Previously the authors reported their results of investigations on the resistivity of molten metals(i), in this paper the resistivity of molten alloys were investigated experimentally and theoretically.

Solid alloys are generally formed from solid solutions and intermetallic compounds, but when they are melted, crystals of these phases are destroyed and atoms are arranged disorderly so that uniform density distribution is formed. It has been clarified(2) that the magnetic susceptibility of molten alloys is given by the sum of the diamagnetic susceptibility contributed by ion cores of alloying atoms and the paramagnetic susceptibility by the free electron gas of valence electrons of atoms. It, therefore, can be assumed that in a molten alloy ions with a respective electric charge equivalent to the atomic valency are generally distributed randomly in an atmosphere of free electron gas, while fluctuations in density distribution of ions and in their concentration distribution occur due to their thermal motions. It is considered that these fluctuations contribute to the resistivity of molten alloys. From the above viewpoint the concentration dependence of the resistivity of molten alloys was mainly studied.

\section{Thermodynamical Properties of Molten Alloys and Concentration Fluctuation}

It is necessary to know the thermodynamical properties of molten alloys to discuss their resistivities,

** The Research Institute for Iron, Steel and Other Metals, Tohoku University, Katahira-cho, Sendai, Japan.

* This paper was read at the Spring Meeting of the Japan Institute of Metals held 3, April, 1961. Tokyo, Japan.

(1) S. Takeuchi and H. Endo: Trans. JIM, 2 (1961), 189.

(2) S. Takeuchi and H. Endo : Trans. JIM, 2 (1961), 188. and many molten alloys, which have been measured, are considered to be a regular solution. So alloys discussed here are confined to those that can be treated as a regular solution.

Let $x$ be a concentration of one of the component atoms in a regular solution of an alloy, then chemical potentials of component atoms $\mathrm{A}$ and $\mathrm{B}$ are given by

$$
\left.\begin{array}{l}
\mu_{\mathrm{A}}=\mu_{\mathrm{A}}^{\circ}+k T \log x+W_{\mathrm{AB}}(1-x)^{2} \\
\mu_{\mathrm{B}}=\mu_{\mathrm{B}}^{\circ}+k T \log (1-x)+W_{\mathrm{AB}} x^{2}
\end{array}\right\},
$$

where $W_{\mathrm{AB}}$ denotes the heat of mixing, $\mu_{\mathrm{A}}^{0}$ and $\mu_{\mathrm{B}}^{0}$ are the chemical potentials of component atoms $A$ and $B$ respectively and are the functions depending upon temperature only. Since the molten alloy under consideration is homogeneous, it will always be

$$
\frac{W_{\mathrm{AB}}}{k T}<2 .
$$

When a parameter $C_{\mathrm{B}}$ instead of $x$ is defined as follows;

$$
C_{\mathrm{B}}=\frac{1-x}{x}
$$

a fluctuation in concentration distribution $\Delta C_{\mathrm{B}}$ in a small volume $\Delta V$ can be written as follows;

$$
\frac{\Delta C_{\mathrm{B}}}{C_{\mathrm{B}}}=\left(\frac{\bar{N}_{\mathrm{B}}+\Delta N_{\mathrm{B}}}{\overline{N_{\mathrm{B}}}}-\frac{\overline{N_{\mathrm{A}}}+\Delta N_{\mathrm{A}}}{\overline{N_{\mathrm{A}}}}\right),
$$

where $\overline{N_{\mathrm{A}}}$ and $\overline{N_{\mathrm{B}}}$ denote the number of atoms $\mathrm{A}$ and $\mathrm{B}$ in $\Delta V$ in the case of mean distribution respectively, and then it is

$$
\Delta C_{\mathrm{B}}=\frac{\overline{N_{\mathrm{B}}^{-}}}{\bar{N}_{\mathrm{A}}}\left(\frac{\Delta N_{\mathrm{B}}}{N_{\mathrm{B}}^{-}}-\frac{\Delta N_{\mathrm{A}}}{\overline{N_{\mathrm{A}}}}\right) .
$$

There are local higher concentrated portions or local lower concentrated portions even in the case of macroscopically uniform distribution of the concentration. Since the mean square of these concentration fluctua- 
tions contributes to resistivity, it is necessary to calculate $\overline{\Delta C_{\mathrm{B}}^{2}}$, which is given by ${ }^{(3)}$

$$
\overline{\Delta C_{\mathrm{B}}^{2}}=\frac{k T}{N x \Delta V\left(\frac{\partial \mu_{\mathrm{B}}}{\partial C_{\mathrm{B}}}\right)} \geq 0,
$$

where $N$ is the mean number of atoms in the unit volume. When $C_{\mathrm{B}}$ is used instead of $x$ in $\mu_{\mathrm{B}}$ of $\mathrm{Eq}$. (1), it becomes

$$
\begin{aligned}
\frac{\partial \mu_{\mathrm{B}}}{\partial C_{\mathrm{B}}} & =\frac{k T}{C_{\mathrm{B}}\left(1+C_{\mathrm{B}}\right)}-\frac{2 W_{\mathrm{AB}}}{\left(1+C_{\mathrm{B}}\right)^{3}} \\
& =\frac{x^{2}}{1-x} k T-2 W_{\mathrm{AB}} x^{3},
\end{aligned}
$$

therefore,

$$
\overline{\Delta C_{\mathrm{B}}^{2}}=\frac{1}{N \Delta V} \frac{1}{x^{4}}-\frac{x(1-x)}{1-2 \frac{W}{k T} \mathrm{AB}-x(1-x)} \geq 0 \text {. (4) }
$$

Even in the case of a regular solution where $W_{A B}=0$ independent of temperature, that is, even in the case in which distribution of atoms surrounding a central atom is in a uniform concentration independent of the centered atom $\mathrm{A}$ or $\mathrm{B}$, there exists a fluctuation in $\Delta V$ provided an extent of $\Delta V$ is the order of an atomic volume. In such a case we can put

$$
W_{\mathrm{AB}}=0 \text { and } N \cdot \Delta V=1
$$

in Eq. (4), and it becomes

$$
\overline{\Delta C_{\mathrm{B}}^{2}}=\frac{x(1-x)}{x^{4}} \text {. }
$$

\section{Temperature Dependence and Concent- ration Dependence of Resistivity of Molten Alloys}

In a molten alloy consisting of $N x \mathrm{~A}$ atoms with the atomic valency $Z_{\mathrm{A}}$ and $N(1-x)$ B atoms with $Z_{\mathrm{B}}$ in unit volume, $N x$ and $N(1-x)$ of ions of the electric charge $Z_{\mathrm{A}} e$ and $Z_{\mathrm{B}} e$ respectively are randomly arranged and in the thermal motion in an atmosphere of the free electron gas of their valence electrons. When discussing the resistivity due to these distributions of ions and electrons, it is convenient to assume a standard state in which $N$ ions with the mean charge $\overline{Z e}$ are in random arrangement, and the concentration and the density are uniformly distributed. The resistivity for such a standard state can be treated in the same way as the residual resistivity of pure metals described in the previous paper(1). Let $\rho_{0}(x, \bar{Z})$ be the resistivity for the standard state of the alloy. Even in the real state of the molten alloy in which the concentration distribution of ions with a different charge is uniform, a fluctuation in density distribution of ions, that is, in ionic charge density distribution arises from their thermal motion. And also even in the case in which the density distribution is uniform, a concentration fluctuation results in a fluctuation in charge density distribution, because charges $Z_{\mathrm{A}} e$ and $Z_{\mathrm{B}} e$ of respective ions $\mathrm{A}$ and $\mathrm{B}$ are different from the mean charge $\bar{Z}$. Electrons are scattered at these fluctuated portions, and such an electron scattering

(3) A. Münster : Statistische Thermodynamik. Springer, (1956), S. 216. by the fluctuation causes an additive resistivity to the resistivity $\rho_{0}(x, \bar{Z})$ for the standard state. Accordingly, total electric resistivity of molten alloys is given by

$$
\rho(x, T)=\rho_{0}(x, \bar{Z})+\rho_{C}(x, T)+\rho_{\kappa}(x, T),
$$

where $\rho_{\kappa}(x, T)$ is a resistivity due to density fluctuation under an uniform concentration distribution of ions, and $\rho_{C}(x, T)$ a resistivity due to concentration fluctuation under uniform density.

\section{Electric resistivity of molten alloys due to density fluctuation under uniform con- centration distribution}

When the density fluctuation in a small volume $\Delta V$ is $\Delta N_{\mathrm{r}}$, the charge density fluctuation is given by $\Delta N_{V} \cdot Z e$. These local excess charges produce a potential field $\psi$ to the mean field due to the uniform density distribution of ions and $\psi$ is given by

$$
\psi=\frac{\bar{Z} \cdot \Delta N_{V} \cdot e}{\gamma} \exp (-q r),
$$

where the screening constant $q$ is given by

$$
q^{2}=\frac{16 \pi^{2} e^{2} m}{h^{2}}\left(\frac{3 \bar{n}}{\pi}\right)^{1 / 3}
$$

using the electron density $\bar{n}$.

Thus, a contribution of a resistivity due to a density fluctuation $\rho_{\kappa}(x, T)$ is calculated in the same way as in the case for pure metals.

$$
\rho_{\kappa}(x, T)=\frac{16 \pi^{2} e^{2}}{3 m}\left(\frac{m}{h}\right)^{3} \frac{\left(\bar{Z} \cdot \Delta N_{V}\right)^{2}}{(n)^{2} \Delta V} f(y)
$$

Using $\overrightarrow{\Delta N}_{V}^{2}$ instead of $\Delta N_{V}^{2}$ in the above equation, it becomes

$$
\begin{aligned}
\rho_{\kappa}(x, T)= & \frac{16 \pi^{2} e^{2}}{3 m}\left(\frac{m}{h}\right)^{3}\left(\frac{N}{\bar{n}}\right)^{2}(\bar{Z})^{2} \\
& \cdot \kappa(x) \cdot k T \cdot f(\mathrm{y}),
\end{aligned}
$$

where $f(y)$ is the known function depending upon the electron density $\bar{n}$.

\section{Resistivity due to concentration fluctuation under uniform density distribution}

A fluctuation in charge density distribution due to concentration fluctuation $\Delta C_{\mathrm{B}}$ in a small volume $\Delta V$ is

$$
\Delta Z e=\frac{\partial \bar{Z} e}{\partial \bar{C}_{\mathrm{B}}} \Delta C_{\mathrm{B}}
$$

and $\overline{Z e}$ is rewritten, using $C_{\mathrm{B}}$ instead of $x$, as follows;

$$
\begin{aligned}
\bar{Z} e & =\left\{Z_{\mathrm{A}} x+Z_{\mathrm{B}}(1-x)\right\} e \\
& =Z_{\mathrm{A}}\left(\frac{1}{1+C_{\mathrm{B}}}\right) e+Z_{\mathrm{B}}\left(\frac{C_{\mathrm{B}}}{1+C_{\mathrm{B}}}\right) e .
\end{aligned}
$$

Therefore, we have

$$
\Delta Z e=\left(Z_{\mathrm{B}}-Z_{\mathrm{A}}\right) e \frac{\Delta C_{\mathrm{B}}}{\left(1+C_{\mathrm{B}}\right)^{2}}
$$

and the charge density fluctuation in $\Delta V$ is expressed by

$$
\Delta Z e N \Delta V=\left(Z_{\mathrm{B}}-Z_{\mathrm{A}}\right) e \cdot x^{2} N \Delta V \cdot \Delta C_{\mathrm{B}} .
$$

Thus, the contribution of the concentration fluctuation to the resistivity is given by 


$$
\begin{aligned}
\rho_{C}(x, T)= & \frac{16 \pi^{2} e^{2}}{3 m}\left(\frac{m}{h}\right)^{3}\left(\frac{N}{\bar{n}}\right)^{2} \\
& \cdot \frac{\left(Z_{\mathrm{B}}-Z_{\mathrm{A}}\right)^{2} \cdot x^{4} \cdot \Delta C_{\mathrm{B}}^{2}(\Delta V)^{2}}{\Delta V} f(y) .(8)
\end{aligned}
$$

When the mean value $\overline{\Delta C_{\mathrm{B}}^{2}}$ is used instead of $\Delta C_{\mathrm{B}}^{2}$,

$$
\begin{aligned}
\rho_{C}(x, T)= & \frac{16 \pi^{2} e^{2}}{3 m}\left(\frac{m}{h}\right)^{3}\left(\frac{N}{i}\right)^{2} . \\
& \frac{\left(Z_{\mathrm{B}}-Z_{\mathrm{A}}\right)^{2}}{N} \frac{x(1-x)}{1-2 \frac{W_{\mathrm{AB}}}{k} T(1-x)} f(y) .(9)
\end{aligned}
$$

In the above formula we put

$$
W_{\mathrm{AB}}=0 \text { or }\left|\frac{W_{\mathrm{AB}}}{k T} x(1-x)\right| \ll 1,
$$

then it becomes

$$
\begin{aligned}
\rho_{C}(x, T)= & \frac{16 \pi^{2} e^{2}}{3 m}\left(-\frac{m}{h}\right)^{3}\left(\frac{N}{\bar{n}}\right)^{2} \\
& \cdot \frac{\left(Z_{\mathrm{B}}-Z_{\mathrm{A}}\right)^{2}}{N} x(1-x) f(y) .
\end{aligned}
$$

This is an excess resistivity due to uniform mixing of ions with a different charge and is equivalent to those due to concentration fluctuation in an extent of the order of atomic volume. The excess resistivity due to uniform mixing of ions is nearly independent of temperature but is parabolic to the concentration. Thus the resistivity of the molten alloy is

$$
\begin{aligned}
\rho(x, T) & =\rho_{o}(x, \bar{Z})+\frac{16 \pi^{2} e^{2}}{3 m}\left(\frac{m}{h}\right)^{3} \\
& \cdot\left[\kappa(x) \cdot k T+\left(\frac{N}{\bar{n}}\right)^{2} \frac{\left(Z_{\mathrm{B}}-Z_{\mathrm{A}}\right)^{2}}{N}\right. \\
& \left.\cdot \frac{x(1-x)}{1-2 \frac{W_{\mathrm{AB}}}{k} T^{-x}(1-x)}\right] f(y),
\end{aligned}
$$

or

$$
\begin{aligned}
\rho(x, T) & =\rho,(x, \bar{Z})+\frac{16 \pi^{2} e^{2}}{3 m}\left(\frac{m}{h}\right)^{3}\left(\frac{N}{\bar{n}}\right)^{2} \\
& \cdot \frac{\left(Z_{\mathrm{B}}-Z_{\mathrm{A}}\right)^{2}}{N} x(1-x) \cdot f(y)+\frac{16 \pi^{2} e^{2}}{3 m} \\
& \cdot\left(\frac{m}{h}\right)^{3}\left[\kappa(x) \cdot k T+\left(\frac{N}{\bar{n}}\right)^{2} \frac{\left(Z_{\mathrm{B}}-Z_{\mathrm{A}}\right)^{2}}{N}\right. \\
& \left.\cdot \frac{2 \frac{W_{\mathrm{AB}}}{k T} x(1-x)}{1-2 \frac{W_{\mathrm{AB}}}{k T} x(1-x)}\right] f(y) .
\end{aligned}
$$

The sum of the first and the second term in the right-hand side of Eq. (12) is the resistivity in the case when atoms with different valencies are randomly distributed in uniform density and uniform concentration, and the third and the forth terms are resistivities due to density and concentration fluctuations, respectively, which are produced by the thermal motion and the existence of the heat of mixing.

\section{Comparison of Theoretical Results with Observations}

The theoretical formula (12) for the resistivity of binary molten alloys as a function of temperature and concentration was experimentally examined.

\section{Change in the resistivity of molten metals due to the addition of a small amount of other elements}

First, when a small amount of various kinds of other metals with different atomic valencies is added to the mother metal $\mathrm{A}$, changes in the resistivity and in the temperature coefficient of resistivity of the molten metal A are discussed. In this case the compressibility $\kappa(x)$ of such a dilute alloy is nearly equal to that of the pure metal $\mathrm{A}$ and only the term $\rho_{C}(x, T)$ in Eq. (11) varies sensitively by the concentration change. Accordingly the change in the resistivity $\Delta \rho$ of the metal A by the addition of one atomic percent of metal $\mathrm{B}$, is given by

$$
\begin{gathered}
\Delta \rho=0.01 \frac{16 \pi^{2} e^{2}}{3 \mathrm{~m}}\left(\frac{m}{h}\right)^{3} \frac{M_{\mathrm{A}}}{d_{\mathrm{A}}} \frac{1}{N_{0}} \\
\cdot \frac{\left(Z_{\mathrm{B}}-Z_{\mathrm{A}}\right)^{2}}{Z_{\mathrm{A}}^{2}} f\left(y_{\mathrm{A}}\right),
\end{gathered}
$$

since we have a relation

$$
\left(\frac{N}{\dot{n}}\right)^{2} \frac{1}{N} \fallingdotseq \frac{1}{Z_{\mathrm{A}}^{2}} \frac{1}{\frac{d_{\mathrm{A}}}{M_{\mathrm{A}}} \cdot N_{0}},
$$

where $d_{\mathrm{A}}$ and $M_{\mathrm{A}}$ are the density and atomic weight of metal A, respectively and $N_{0}$ is Avogadros' number. Change of resistivity in this case is proportional to $\left(Z_{\mathrm{A}}-Z_{\mathrm{B}}\right)^{2}$, so Linde's law, which is known for solid solutions, is expected to hold also in the case of the molten alloys. The temperature dependence of the 3rd term in the right hand side of Eq. (12) is negligibly small, because it is proportional to $x^{2}(1-x)^{2}$, and the compressibility $\kappa(x)$ varies very slightly with temperature, so the temperature coefficient $d \rho / d T$ becomes

$$
\frac{d \rho}{d T}=\frac{16 \pi^{2} e^{2}}{3 m}\left(\frac{m}{h}\right)^{3} \kappa_{\mathrm{A}} \cdot k \cdot f\left(y_{\mathrm{A}}\right),
$$

which is equal to the temperature coefficient of the resistivity of the pure metal A. Mathiesen's law for the solid solution is expected to hold also in the molten alloy.

Changes in the resistivity of the following pure metals, $\mathrm{Bi}, \mathrm{Sn}$, and $\mathrm{Zn}$ were measured in cases when they were alloyed by the addition of one atomic percent of other metals with different atomic valencies. In Fig. 1. the increase in the resistivity $\Delta \rho$ of molten $\mathrm{Bi}$ is plotted against the square of the valency difference $\left(Z_{\mathrm{A}}-Z_{\mathrm{B}}\right)^{\prime}$ ' when one atomic percent of $\mathrm{Ag}, \mathrm{Cd}$, $\mathrm{In}, \mathrm{Sn}$ and $\mathrm{Pb}$ is added respectively, and $\Delta \rho$ of molten tin due to addition of $\mathrm{Ag}, \mathrm{Zn}$ and $\mathrm{Al}$ is also plotted. From the figure it is clear that each $\Delta \rho$ is in a linear relation to $\left(Z_{\mathrm{A}}-Z_{\mathrm{B}}\right)^{2}$ and the observed $\Delta \rho$ agrees with the calculated straight line. It was shown that $d \rho / d T$ of the dilute alloy of tin containing a small concentration of $\mathrm{Bi}, \mathrm{Pb}, \mathrm{Al}, \mathrm{Zn}$ and $\mathrm{Ag}$ is equal nearly to that of the pure metal tin. However, the effect of addition of other elements to the molten metal $\mathrm{Zn}$ seems to be so complicated that the linear relation between $\Delta \rho$ and $\left(Z_{\mathrm{A}}-Z_{\mathrm{B}}\right)^{2}$ does not hold, and $d \rho / d T$ in dilute molten alloys of zinc differs a little from that of the pure molten zinc, and the same phenomena are also expected in the other divalent metals, $\mathrm{Mg}$ and 
$\mathrm{Cd}$. The abnormality in the temperature dependence of resistivity of the divalent metals $\mathrm{Zn}$ and $\mathrm{Cd}$ has been reported in the previous paper. ${ }^{(1)}$
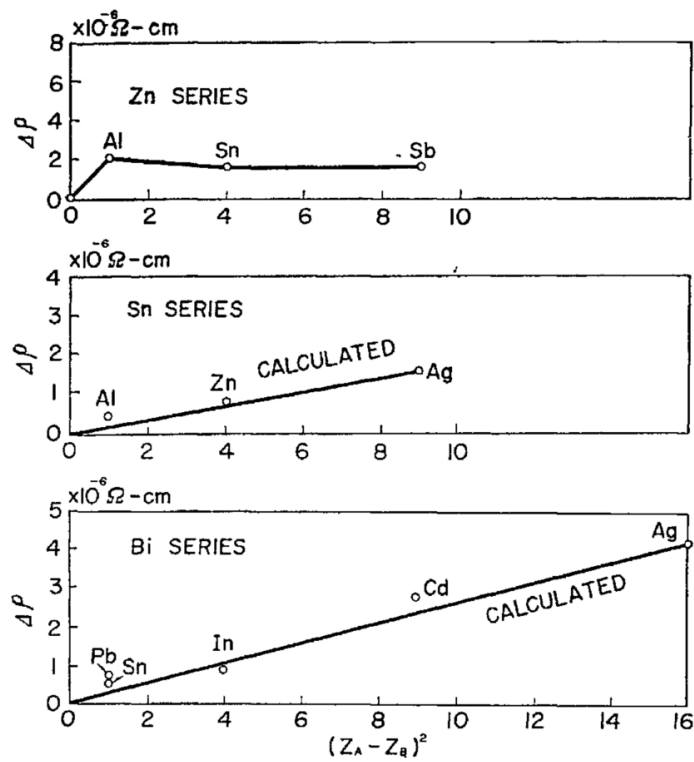

Fig. 1 Changes in resistivity of molten metals due to addition of 1 atomic percent of other metals, plotted against the square of difference in atomic valencies.

2. The resistivity of binary molten alloys consisting of component metals having the same atomic valencies

In the case of $Z_{\mathrm{A}}=Z_{\mathrm{B}}$, we obtain from Eq. (12)

$$
\begin{aligned}
\rho(x, T) & =\rho_{o}(x, \bar{Z}) \\
& +\frac{16 \pi^{2} e^{2}}{3 m}\left(\frac{m}{h}\right)^{3} \kappa(x) \cdot k T \cdot f(y) .
\end{aligned}
$$

$\rho_{0}(x, \bar{Z})$ is the resistivity for the standard state in which ions with the mean charge $Z e$ are distributed in an uniform density and uniform concentration, so it is presumed that the relation between $\rho_{0}(x, \bar{Z})$ and concentration is described approximately by a straight line connecting $\rho_{0}^{\mathrm{A}}$ and $\rho_{0}^{\mathrm{B}}$ which are obtained by extrapolation of high temperature resistivities of pure metals A and B to absolute zero of temperature, respectively, but there exists a little deviation from such a straight line according to the difference between atomic radii or densities of both metals on which the cross section of electron scattering depends.

In the alloys, $\mathrm{Pb}-\mathrm{Sn}$ and $\mathrm{Sb}-\mathrm{Bi}$, of constituent atoms with the same valency $Z_{\mathrm{B}}=Z_{\mathrm{A}}$, the observed $\rho_{0}(x)$ versus concentration curves, which were obtained by extrapolation of their high temperature resistivities to $0^{\circ} \mathrm{K}$, are nearly linear, respectively, as shown in Fig. 2. The concentration dependence of the second term in Eq. (15) is due to $f(y)$ and $\kappa(x)$. But a change in $y$ and hence a change in $f(y)$ with varying concentration is negligibly small, so far as the atomic radii of the component metals with the same valency in the above alloys do not differ markedly from each other. And any concentration dependence of the compressibility of the alloys has not yet been measured, however, in the case of $Z_{\mathrm{A}}=Z_{\mathrm{B}}$, the following relation

$$
\kappa(x)=\kappa_{\mathrm{A}} \cdot x+\kappa_{\mathrm{B}}(1-x)
$$

can be assumed to hold approximately as long as: difference of the atomic radii or the ionic radii of alloying elements is so small. In Eq. (16) $\kappa_{\mathrm{A}}$ and $\kappa_{\mathrm{B}}$

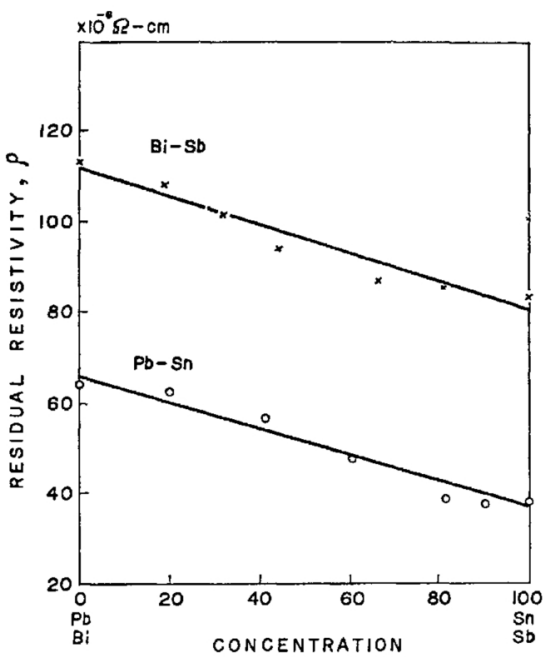

Fig. 2 Concentration dependence of residual resistivity of molten alloys, $\mathrm{Pb}-\mathrm{Sn}$ and $\mathrm{Bi}-\mathrm{Sb}$.

are the compressibilities of pure metals $A$ and $B$ respectively. Therefore, in the case of $Z_{\mathrm{A}}=Z_{\mathrm{B}}$, it is. expected that concentration dependence of the resistivity of alloys is nearly linear as seen from Eq. (15) when the above relation (16) holds, and the temperature coefficient $d \rho / d T$ is also nearly in a linear relation to. concentration.

The plots of the observed resistivities versus concentration of the alloys, $\mathrm{Bi}-\mathrm{Sb}$ and $\mathrm{Pb}-\mathrm{Sn}$, are shown in Fig. 3 and Fig. 4 respectively, and these agree approximately with the straight line connecting the
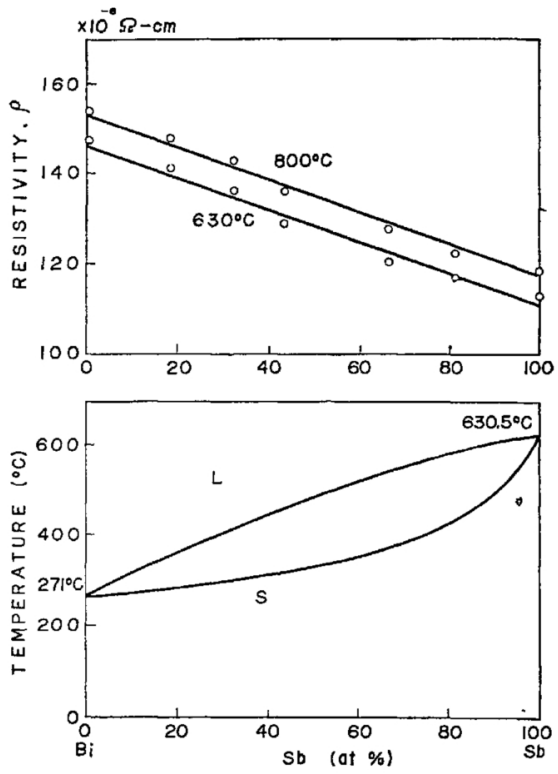

Fig. 3 Plots of resistivity versus concentration of the molten alloy $\mathrm{Bi}-\mathrm{Sb}$ at $630^{\circ} \mathrm{C}$ and $800^{\circ} \mathrm{C}$, and the phase diagram of the alloy.

resistivities of the constituent metals of the respective alloys. And also the plots of $d \rho / d T$ versus concentration in the above alloys shown in Fig. 5 are in close agreement with the straight lines connecting those of the constituent metals respectively, as expected. theoretically. 
3. The resistivity of molten alloys of constituent metals with different valencies

In the case of $Z_{\mathrm{A}} \neq Z_{\mathrm{B}}$, the temperature dependence

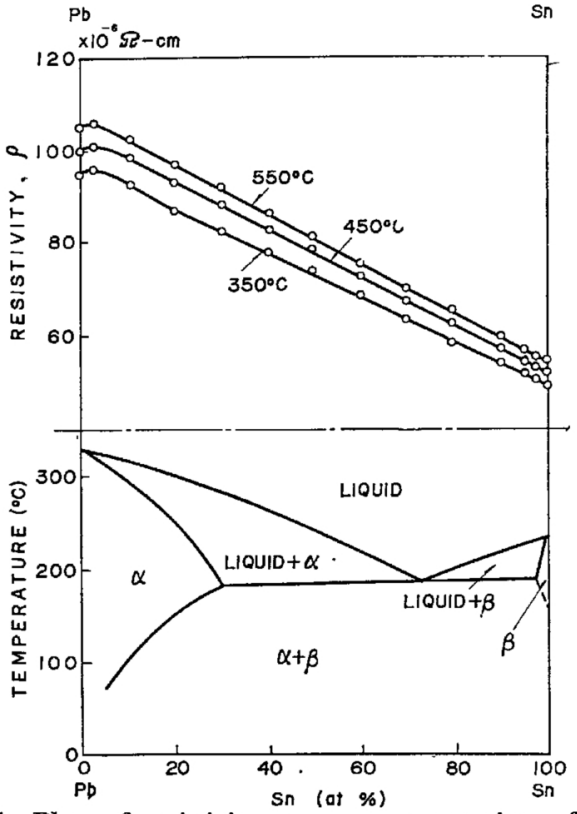

Fig. 4 Plots of resistivity versus concentration of the molten alloy $\mathrm{Pb}-\mathrm{Sn}$ at $350^{\circ}, 450^{\circ}$ and $550^{\circ} \mathrm{C}$ and the phase diagram of the alloy.

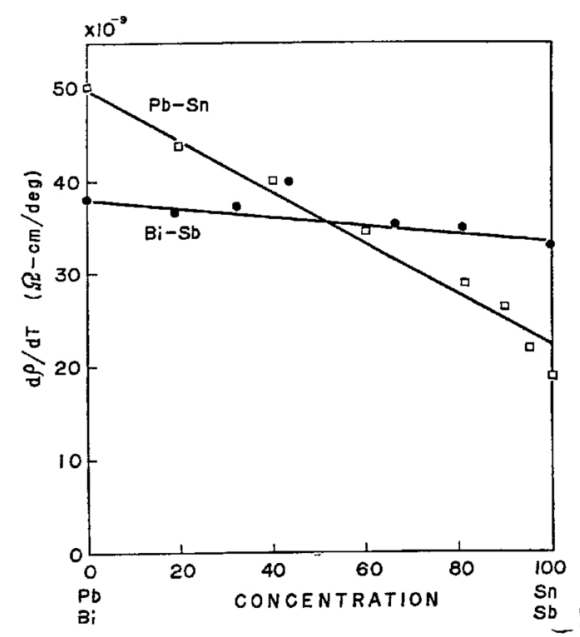

Fig. 5 Varition of temperature coefficient of resistivity with change of concentration in molten alloys $\mathrm{Pb}-\mathrm{Sn}$ and $\mathrm{Bi}-\mathrm{Sb}$.

as well as the concentration dependence of the resistivity of molten alloys are influenced markedly by the magnitude and the sign of heat of mixing $W_{\mathrm{AB}}$. First, the case of the uniform concentration distribution,

$$
W_{\mathrm{AB}}=0 \text { or }\left|\frac{W_{\mathrm{AB}}}{k T} x(1-x)\right| \ll 1
$$

is discussed. From Eq. (12) it follows :

$$
\begin{aligned}
\rho(x, T) & =\rho_{o}(x, \bar{Z})+\frac{16 \pi^{2} e^{2}}{3 m}\left(\frac{m}{h}\right)^{3} \\
& \cdot \frac{\left(Z_{\mathrm{B}}-Z_{\mathrm{A}}\right)^{2}}{N} x(1-x) \cdot f(y) \\
& +\frac{16 \pi^{2} e^{2}}{3 m}\left(\frac{m}{h}\right)^{3} \cdot \kappa(x) \cdot k T \cdot f(y) .
\end{aligned}
$$

The first and second terms of the right hand side of
Eq. (17) are independent of temperature, so the temperature dependence of the resistivity is given by the third term. When the compressibility $\kappa(x)$ depends upon temperature very slightly, the resistivity is expected to be in nearly a linear relation to temperature. As described above, any measurement of the compressibility for many molten alloys has not yet been preformed. However, it is expected that if the measured plot of $d o / d T$ versus concentration of an alloy is linear, the compressibility of the alloy is followed by Eq. (16), and if the plot becomes-a curve, the compressibility is related to the concentration in a non-linear form different from Eq. (16).

The residual resistivity, independent of temperature, is given by the sum of the first and second terms. The first term $\rho_{0}(x, Z)$ is considered to be roughly in a linear relation to concentration, so the concentration dependence of the residue is mainly given by the second term, being in a parabolic form having a maximum near fifty percent of concentration, as seen from Eq. (17). Therefore, the concentration dependence of resistivity of molten alloys is given by the sum of the parabolic function of the residual resistivity and the third term $\rho_{\varepsilon}(x, T)$, which depends upon the compressibility of alloys.

Since the observed resistivities of the alloys, Bi-Sn and $\mathrm{Bi}$-In depend linearly on temperature, $d \rho / d T$ for these alloys can be easily obtained experimentally in the function of concentration. The plot of $d \rho / d T$ versus concentration is shown in Fig. 6, in which in a $\mathrm{Bi}-\mathrm{Sn}$ alloy, it is concave and in a Bi-In alloy it is convex. $\rho_{o}(x, \bar{Z})$ is obtained by subtracting the calculated values of $\rho_{\mathrm{C}}(x)$ for each composition from the

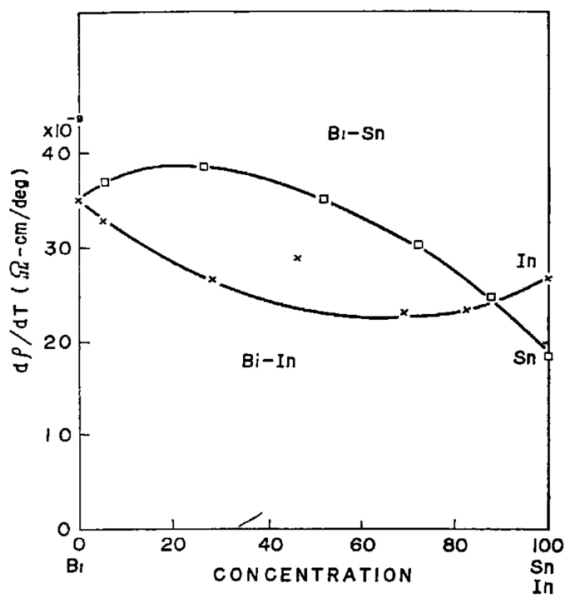

Fig. 6 Relations between temperature coefficients of resistivity and alloy concentrations in binary molten alloys, $\mathrm{Bi}-\mathrm{Sn}$ and $\mathrm{Bi}-\mathrm{In}$.

residual resistivity which is obtained by extrapolation of the high temperature resistivities to $0^{\circ} \mathrm{K} . \rho_{o}(x, \bar{Z})$ estimated above, as shown in Fig. 7, deviates slightly from a linearity in both alloys. The observed resistivities of the alloys $\mathrm{Bi}$-In and $\mathrm{Bi}-\mathrm{Sn}$ are plotted against each composition in Figs. 8 and 9, respectively. All of these curves are nearly parabolic. In Fig. 10 a difference $\Delta \rho$ (obs) between the observed resistivity curve in Fig. 8 and in Fig. 9 and a line connecting the resistivities of the component metals is shown. The 
$\Delta \rho$ (obs) thus obtained is nearly a parabolic curve for each of the two alloys as seen in Fig. 10, and this curve agrees approximately with the theoretical curve which is the sum of the calculated curve of $\rho_{\mathrm{c}}(x)$ and

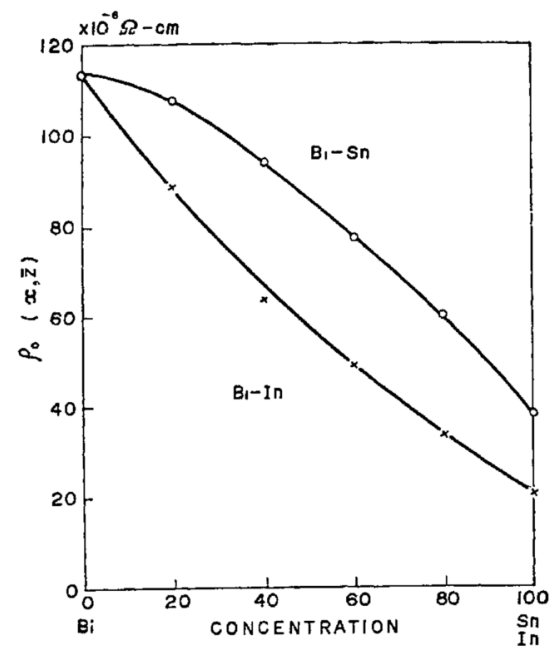

Fig. 7 Concentration dependences of resistivities of the standard state in moltten alloys, Bi-Sn and Bi-In.

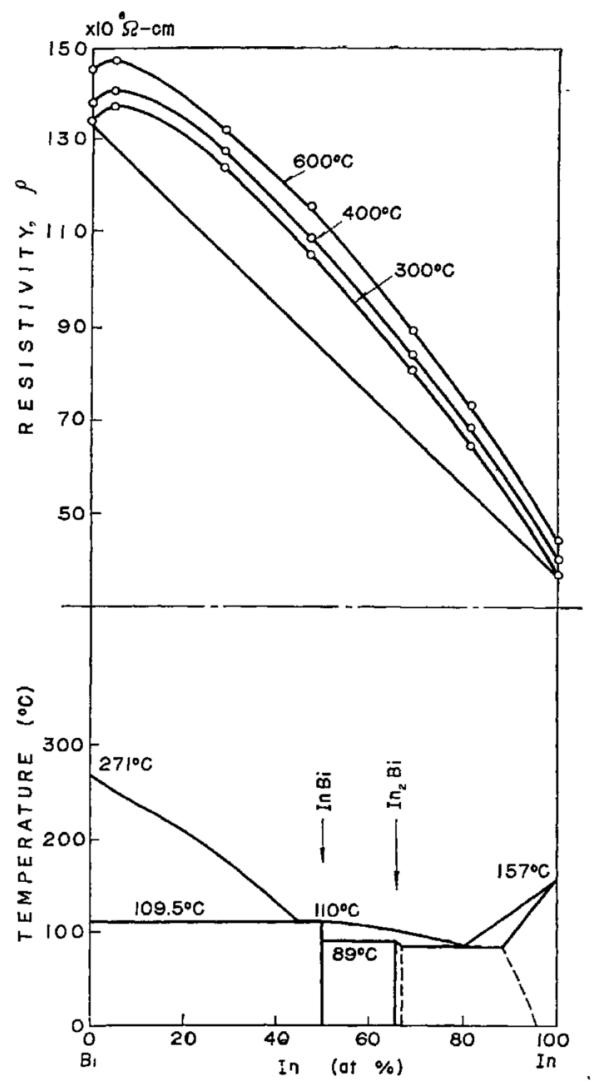

Fig. 8 Plots of resistivity versus concentration of the molten alloy $\mathrm{Bi}$-In at $300^{\circ}, 400^{\circ}$ and $600^{\circ} \mathrm{C}$ and the phase diagram ot the alloy.

that of $\rho_{K}(x, T)$ obtained from the plot of $d \rho / d T$ versus concentration in Fig. 6.

The heat of mixing of a Bi-Sn alloy is $+161 \mathrm{cal} /$ $\mathrm{mol}^{(4)}$ at the composition of $51.8 \% \mathrm{Bi}$ at $350^{\circ} \mathrm{C}$, which corresponds to

$$
W_{\mathrm{AB}} x(1-x)=-161 \mathrm{cal} / \mathrm{mol} \text { at } x=0.5 .
$$

Therefore the condition

(4) M. Kawakami : Sci. Rep Tohoku University, 16 (1927), 915.

$$
\left|\frac{W_{\mathrm{AB}}}{k T} x(1-x)\right| \ll 1
$$

is satisfied in this alloy.

When the term containing the heat of mixing $W_{A B}$

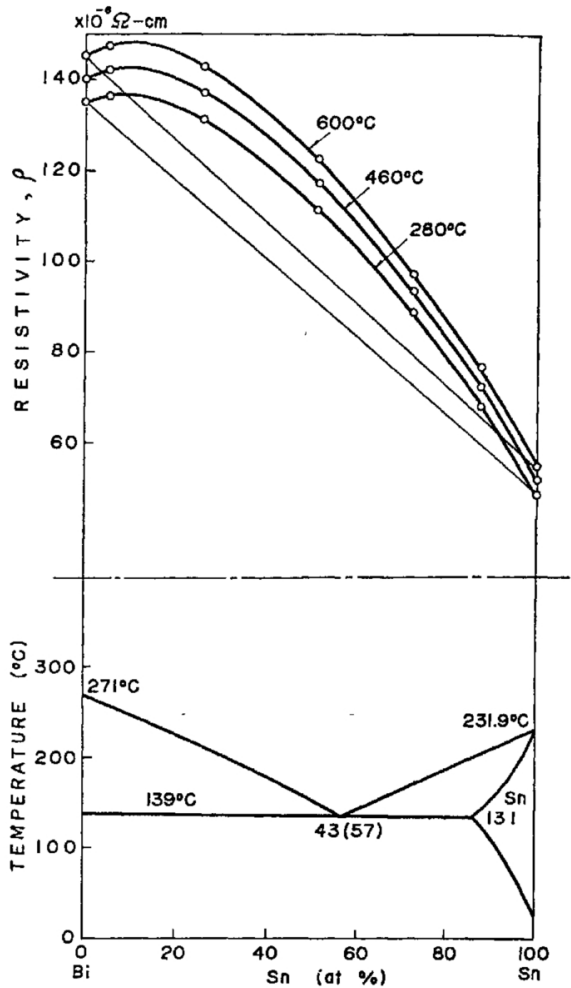

Fig. 9 Plots of resistivity versus concentration of the molten alloy at $280^{\circ}, 460^{\circ}$ and $600^{\circ} \mathrm{C}$ and the phase diagram of the alloy.
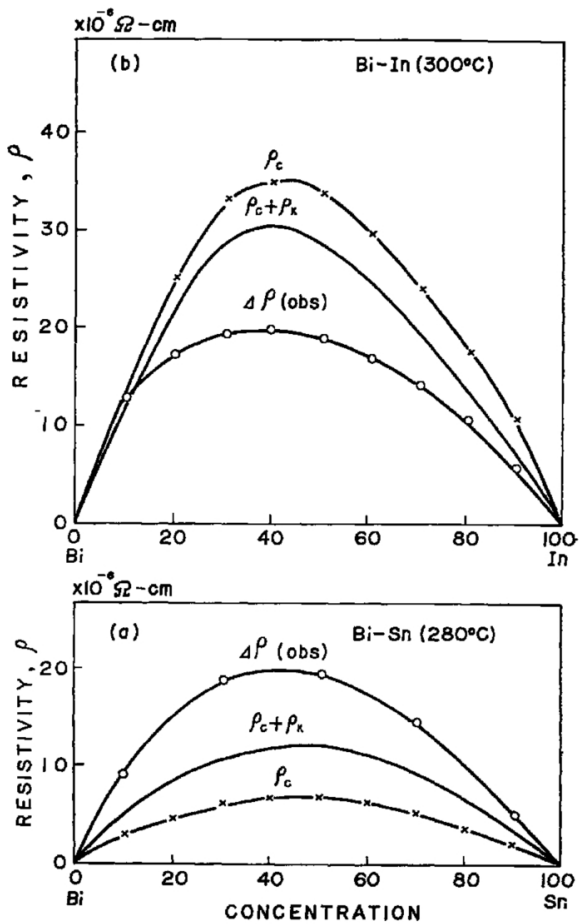

Fig. 10 Contributions of concentration fluctuation and density fluctuation to the resistivity of the molten alloys, $\mathrm{Bi}-\mathrm{In}$ and $\mathrm{Bi}-\mathrm{Sn}$.

can not be negligible in Eq. (11) or (12) because of a large heat of mixing, the resistivity varies complicatedly with changes in temperature and in concentration, and it can be shown that there is a concentration range where the resistivity becomes smaller with increasing. 
temperature in the case of $W_{\mathrm{AB}}>0$. The above will be described in detail in a later report.

\section{Summary}

Electric resistivity for some binary molten alloys were examined experimentally and theoretically.

1. Based on the assumption that in a molten state of alloys valency electrons of atoms behave like free electrons and ions with electric charges corresponding to the atomic valency are randomly distributed, and that density fluctuations and concentration fluctuations occur in these ionic arrangements due to the thermal agitation, temperature dependence and concentration dependence of the resistivity of molten alloys were calculated as the sum of a term contributed by density flutuation and a term by concentration fluctuation.

2. An increase in a resistivity $\Delta \rho$ of a molten pure metal $A$ by addition of a small amount of an other metal $\mathrm{B}$ is proportional to $\left(Z_{\mathrm{A}}-Z_{\mathrm{B}}\right)^{2}$ in which $Z_{\mathrm{A}}$ and $Z_{\mathrm{B}}$ are atomic valencies of the base metal $A$ and metals $\mathrm{B}$, and a temperature coefficent of the resistivity $\frac{d \rho}{d T}$ of the base metal is not influenced by the addition of small amounts of other metals. These theoretical results were confirmed experimentally in some molten metals. However, it was found that when the divalent metals were used as the base metals, the above theoretical results were not satisfied.

3. In an alloy of component metals with the same atomic valency, the resistivity of the alloy varies linearly with concentration provided the compressibility of the alloys is given by a linear function of concentration. It was shown that such a linear relation was satisfied experimentally in the alloys $\mathrm{Bi}-\mathrm{Sb}$ and $\mathrm{Pb}-\mathrm{Sn}$.

4. In alloys of component metals with different atomic valencies, the resistivity varies linearly with increasing temperature and parabolically with changing concentration, provided the heat of mixing of the alloy is as small as

$$
\left|\frac{W_{\mathrm{AB}}}{k T} x(1-x)\right| \ll 1 .
$$

The measured resistivities of the alloys $\mathrm{Bi}-\mathrm{In}$ and $\mathrm{Bi}$ Sn were shown to satisfy the linear relation to temperature and the parabolic relation to concentration. 